\title{
On redundancy-modified NAND multiplexing
}

\author{
DIAO Ming ${ }^{1}$, YU Lianhua ${ }^{1, *}$, and CHEN Xiaobo ${ }^{2}$ \\ 1. College of Information and Communication Engineering, Harbin Engineering University, Harbin 150001, China; \\ 2. College of Automation, Harbin Engineering University, Harbin 150001, China
}

\begin{abstract}
In order to make systems that are based on unreliable components reliable, the design of fault tolerant architectures will be necessary. Inspired by von Neumann's negative AND (NAND) multiplexing and William's interwoven redundant logic, this paper presents a fault tolerant technique based on redundancy-modified NAND gates for future nanocomputers. Bifurcation theory is used to analyze fault tolerant ability of the system and the simulation results show that the new system has a much higher fault tolerant ability than the conventional multiplexing based on NAND gates. According to the evaluation, the proposed architecture can tolerate a device error rate of up to $10^{-1}$, with multiple redundant components. This fault tolerant technique is potentially useful for future nanoelectronics.
\end{abstract}

Keywords: fault tolerant, multiplexing, redundancy-modified negative AND (NAND) gate.

DOI: $10.21629 / J S E E .2018 .04 .21$

\section{Introduction}

With the rise of nanoelectronic technology, we are inevitably faced with the question of how to build reliable systems out of unreliable components. To tackle this problem, several fault tolerant techniques based on redundancy have been investigated, such as N-tuple modular redundancy (e.g., triple modular redundancy) [1-3], reconfiguration [3-6], quadded logic [7,8] and other redundancy techniques [9-14]. However, with these techniques alone, high fault tolerance is hard to achieve for nanocomputers since faulty components are pervasive in space and time. Thus, von Neumann's multiplexing, which essentially treats unreliable components as an integral part of the system, has received attention [15]. In 1952, von Neumann initiated the study of multiplexing technique [16], since then, a wealth of papers reporting performance analysis of multiplexing have been published. The multiplexing technique theoretically demonstrated may work

\footnotetext{
Manuscript received December 06, 2016.

*Corresponding author.

This work was supported by the National Natural Science Foundation of China (61571149).
}

effectively with a practically acceptable redundancy overhead [17] and has been studied as an effective fault tolerant technique for protection against the increasing transient faults in nanoelectronic circuits [15,18-22]. The vast majority of those studies have focused on negative AND (NAND) multiplexing [18-23], majority multiplexing [24-26] and negative OR (NOR) multiplexing [27], where NAND multiplexing obtained the most extensive attention.

However, the traditional NAND multiplexing architecture can only tolerate a device error rate of up to $10^{-2}$, in other word, its fault tolerant ability is not very satisfactory. In this paper, instead of the NAND gate which is used for two-layer error correction, we adopt the redundancymodified NAND gate [28] which is used for single-layer error correction to build restorative units. As a result, the traditional NAND multiplexing has been improved to have a much better fault tolerant ability. And different from other papers, here, we focus our study on the exclusive OR (XOR) multiplexing, which was presented for the first time in our last work [29]. The improved multiplexing architecture consists of XOR gates and redundancy-modified NAND gates and comprises an executive unit and restorative units. Restorative units perform the error correction function, and they are composed of redundancy-modified NAND gates. The system performance of the architecture is evaluated by studying its fault tolerant ability, i.e., gate error threshold and input signal error threshold, where the gate error threshold is the maximum gate error probability that the system can still work properly, and the input signal error threshold is the maximum input signal error probability that the system can tolerate. Multiplexing based on redundancy-modified NAND has a much better fault tolerant ability than traditional multiplexing based on NAND gates. XOR multiplexing system has a unique feature, we name it as critical point property, which can indicate the fault tolerant ability of the system.

The rest of this paper is arranged as follows. In Section 2, the error distributions in executive unit and redundancy-modified NAND multiplexing system are pre- 
sented. In Section 3, we discuss the bifurcation analysis which is followed by Section 4: fault tolerant ability analysis of redundancy-modified NAND multiplexing system. And Section 5 is the conclusion.

\section{Redundancy-modified NAND multiplexing technology}

The multiplexing technique uses redundant components to obtain reliable synthesis from unreliable components. To realize this technology, two types of units are usually required: they are executive unit and restorative unit.

\subsection{An XOR multiplexing unit}

The executive unit of redundancy-modified NAND multiplexing could be XOR, exclusive negative OR (XNOR), NOR or NAND, and here, we choose the XOR unit as the executive unit to analyze redundancy-modified NAND multiplexing. As shown in Fig. 1, the XOR multiplexing unit has the same structure with the NAND multiplexing unit. Consider an XOR gate with an error probability $\varepsilon$. Duplicate the XOR gate $N$ times, and replace each input of the XOR gate as well as its output by a bundle of $N$ lines. The XOR multiplexing unit takes two bundles of $N$ wires as inputs and generates a bundle of $N$ wires as outputs. $X$, $Y$ and $Z$ are the inputs and output of the multiplexing unit, respectively.

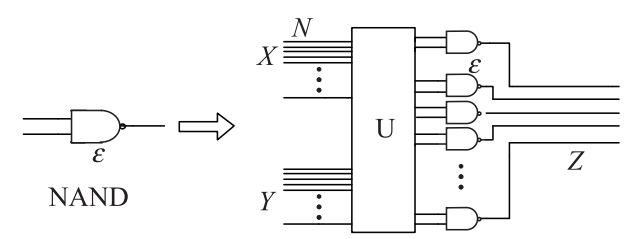

(a) NAND

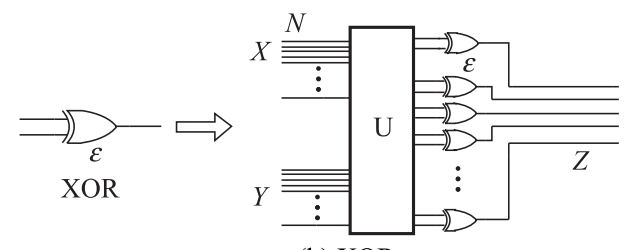

(b) XOR

Fig. 1 NAND and XOR multiplexing units

The randomizing unit $\mathrm{U}$ performs random permutation. Despite this operation, the inputs from the first bundle are randomly paired with those in the second bundle to form the input pairs to the duplicated XOR gates [19].

In systems based on this construction, each signal is carried on a bundle of $N$ wires instead of a single wire and every logic computation is done by $N$ duplicated gates simultaneously. Assume that the error probability $\varepsilon$ is a constant and the type of fault the XOR gate makes is that it inverts its output; i.e., acts as an XNOR gate (a von Neumann error).

\subsection{Error distribution in XOR multiplexing unit}

The XOR multiplexing unit is shown in Fig. 1(b). Assume that $(x, y, z)$ are relative levels of excitation of the two input bundles and of the output bundle, respectively. If the error probabilities in the two input wires are independent, the probability of the output being stimulated is $z=x(1-y)+y(1-x)$ (assume that the XOR gate is fault free). If each gate has a probability $\varepsilon$ of making a von Neumann error, the probability of its output is found stimulated as

$$
\begin{gathered}
z=(1-\varepsilon)[x(1-y)+y[1-x)]+ \\
\varepsilon[x y+(1-x)(1-y)]
\end{gathered}
$$

And the probability of its output being non-stimulated is $1-z$. If the $N$ XOR gates function independently, the entire XOR multiplexing unit constitutes a Bernoulli sequence. The distribution of the probability of the stimulated output is, therefore, the binomial distribution, and the probability of exactly $k$ outputs being stimulated is then

$$
P(k)=\left(\begin{array}{c}
N \\
k
\end{array}\right) z^{k}(1-z)^{N-k} .
$$

And when $N$ is rather large $(N>1000)$, the probability density of $k$ can be shown as

$$
f(k)=\frac{1}{\sqrt{2 \pi N z(1-z)}} \mathrm{e}^{-\frac{1}{2}((k-N z) / \sqrt{N z(1-z)})^{2}} .
$$

Therefore, the error probability of the XOR multiplexing unit is approximately normally distributed when $N$ is rather large $(N>1000)$ [19].

\subsection{Redundancy-modified NAND restoring organs}

If we assume that the two input bundles of the executive unit have almost the same stimulated or non-stimulated levels (which is likely in circuits), then for XOR, the error probability of the output bundle will always be larger than the error probability in either one of the input bundles. Hence, we need a unit to restore the original stimulated level of the executive unit and this unit is the restorative unit, also known as the restoring organ. Any logic gates, like NAND gate, NOR gate, AND-OR gates, effectively alternate critical errors and subcritical errors, thereby performing error correction and could be used as restoring organs. Note that an error is critical if its occurrence on one of the inputs causes an incorrect output, while a subcritical error is any error which is not critical error. There are different types of restoring organs. Two layers restoring organ that uses two inputs NAND gates can be found in von Neumann's NAND multiplexing [15,16,19-21], but here, instead of two layers organs, we use a single layer restoring organ to realize it. This single layer restoring 
organ consists of four inputs AND-OR-NOT gate, which is also called redundancy-modified NAND gate [28]. The redundancy-modified NAND gate corrects all single error. Its notation is shown in Fig. 2.

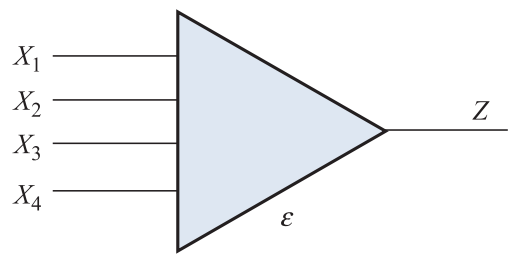

Fig. 2 Notation of AND-OR-NOT gate

If four redundant signals $x_{1}, x_{2}, x_{3}, x_{4}$ are used as inputs to this gate, the circuit can be arranged so that the output is

$$
z=\overline{x_{1} x_{2}+x_{3} x_{4}} .
$$

If none or one of the $x_{i}$ signals is in error, then the output will be the correct value of $z$. Assume that the redundancy-modified NAND gate has the same error probability $\varepsilon$ of making a von Neumann error. Denote the probabilities of the four inputs and output being stimulated by $X_{1}, X_{2}, X_{3}, X_{4}$ and $Z$, respectively. And further assume that the four inputs are independent. Then the probability of the output of redundancy-modified NAND gate being stimulated is

$$
\begin{aligned}
Z= & (1-\varepsilon)\left[\left(1-X_{1} X_{2}\right)\left(1-X_{3} X_{4}\right)\right]+ \\
& \varepsilon\left[1-\left(1-X_{1} X_{2}\right)\left(1-X_{3} X_{4}\right)\right] .
\end{aligned}
$$

\subsection{Redundancy-modified NAND multiplexing architecture}

Multiplexing system is composed of an executive unit and multiple restoring organs. In order to compare it with others, we consider three multiplexing systems based on three different restoring organs, which are redundancy-modified NAND restoring organ, von Neumann's NAND restoring organ and another two layers restoring organ which uses AND gates and OR gates. One version of the AND-OR restoring organ is shown in Fig. 3. Note that it is also possible to have the OR gates in the first layer and the AND gates in the second, or to use them randomly instead of systematic interconnections.

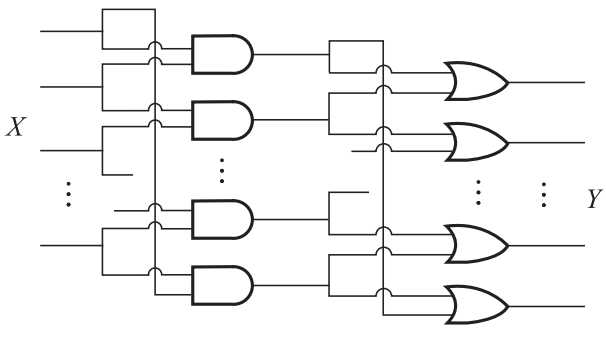

Fig. 3 AND-OR restoring organ
Assume that AND gates and OR gates have the same error probability $\varepsilon$ of making a von Neumann error and the probability of inputs being stimulated is $X$. Then the probability of the outputs being stimulated is

$$
\begin{gathered}
Y=(1-\varepsilon)\left\{1-\left[1-\left[(1-\varepsilon) X^{2}+\varepsilon\left(1-X^{2}\right)\right]^{2}\right]\right\}+ \\
\varepsilon\left[1-\left[(1-\varepsilon) X^{2}+\varepsilon\left(1-X^{2}\right)\right]\right]^{2}
\end{gathered}
$$

Three kinds of XOR multiplexing are shown in Fig. 4, where the XOR multiplexing based on NAND restoring organs was presented in [29] for the first time. For simplicity, below, we use X_NAND represents XOR multiplexing based on NAND restoring organs, X_RMNAND represents XOR multiplexing based on redundancy-modified NAND restoring organs and X_AND-OR represents XOR multiplexing based on AND-OR restoring organs, respectively. In order to make the system stable, multiply restoring organs would be necessary and note that the odd stage number is necessary to keep the XOR function.

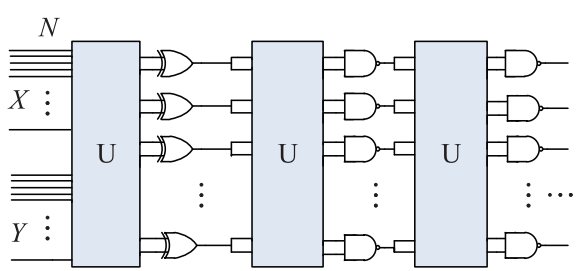

(a) X_NAND

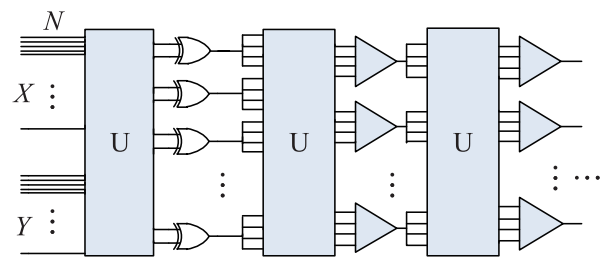

(b) X RMNAND

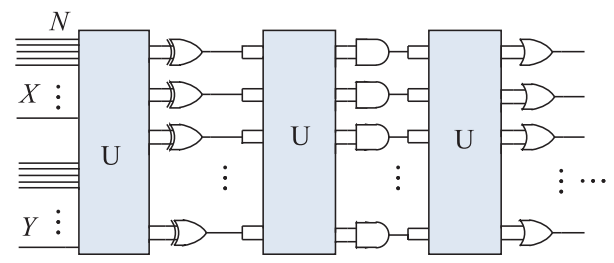

(c) X_AND-OR

Fig. 4 Three kinds of XOR multiplexing system

\section{Bifurcation analysis of redundancy- modified NAND multiplexing system}

In order to gain understanding of the system dynamics of probabilistic logics and the associated concept of reliable computation, here, we focus on the operation of individual unreliable two inputs XOR gates and four inputs 
redundancy-modified NAND gates in a binary tree of cascaded XOR gates and redundancy-modified NAND gates, which is shown in Fig. $5[15,18]$. We assume that the XOR gates and redundancy-modified NAND gates make a von Neumann error with the same probability $\varepsilon$, while the input and output wires function reliably.
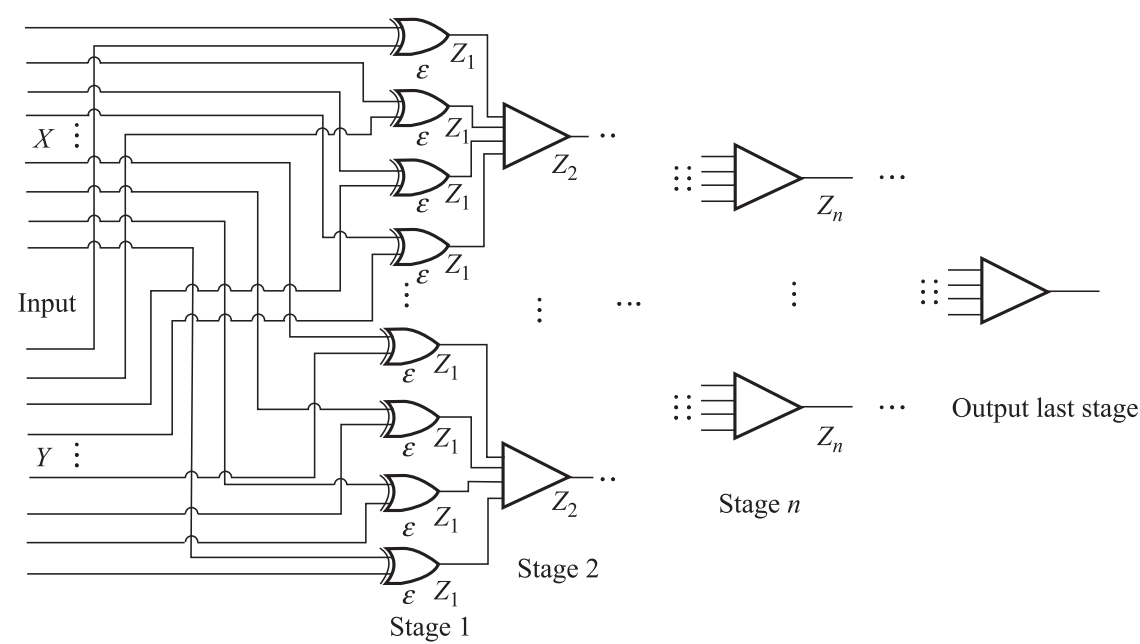

Stage $n$

Fig. 5 Schematic of a full binary tree whose nodes are faulty two inputs XOR gates and redundancy-modified NAND gates with error probability $\varepsilon$

Denote the probabilities of the two inputs of XOR gate being stimulated by $X, Y$ and further assume that the two inputs are independent. Then the probability of the output of the XOR gate being stimulated is

$$
\begin{aligned}
Z_{1}= & (1-\varepsilon)[X(1-Y)+Y(1-X)]+ \\
& \varepsilon[X Y+(1-X)(1-Y)] .
\end{aligned}
$$

Assume that this circuit is a discrete time system and all inputs to the XOR gates are independent and each two inputs of those XOR gates have the same probabilities $X$ and $Y$ being stimulated. This structure guarantees that the inputs to all redundancy-modified NAND gates at an arbitrary stage $n$ are also independent and have same probabilities $Z_{n}$ being stimulated [15]. Then for the second stage, the first stage of redundancy-modified NAND gates, the probabilities of the output being stimulated would be $Z_{2}$ :

$$
Z_{2}=(1-\varepsilon)\left(1-Z_{1}^{2}\right)^{2}+\varepsilon\left[1-\left(1-Z_{1}^{2}\right)^{2}\right] .
$$

For such a construction, (8) reduces to a simple iterative equation

$$
Z_{n+1}=(1-\varepsilon)\left(1-Z_{n}^{2}\right)^{2}+\varepsilon\left[1-\left(1-Z_{n}^{2}\right)^{2}\right] .
$$

Do the same operation to X_AND-OR and X_NAND and their iterative equations are

$$
\begin{gathered}
Z_{n+1}=(1-\varepsilon)\left\{1-\left[1-\left[(1-\varepsilon) Z_{n}^{2}+\varepsilon\left(1-Z_{n}^{2}\right)\right]^{2}\right]\right\}+ \\
\varepsilon\left\{1-\left[(1-\varepsilon) Z_{n}^{2}+\varepsilon\left(1-Z_{n}^{2}\right)\right]\right\}^{2} \\
Z_{n+1}=1-\varepsilon+(2 \varepsilon-1) Z_{n}^{2} .
\end{gathered}
$$

In order to better understand the relationship between system dynamics and gate error probability, we carry out bifurcation analysis of (9), (10) and (11), respectively $[15,18]$. For any $\varepsilon \in[0,1 / 2]$, we choose an initial arbitrary condition $X, Y$ and generate sequence $Z_{i}(i=1,2, \ldots)$, by iterating (9), (10) and (11) until they converge to an attractor. Those attractors for the sequences are then plotted against each $\varepsilon[15,18]$. This leads to the bifurcation diagram in Fig. $6(\Delta \varepsilon=0.005)$.

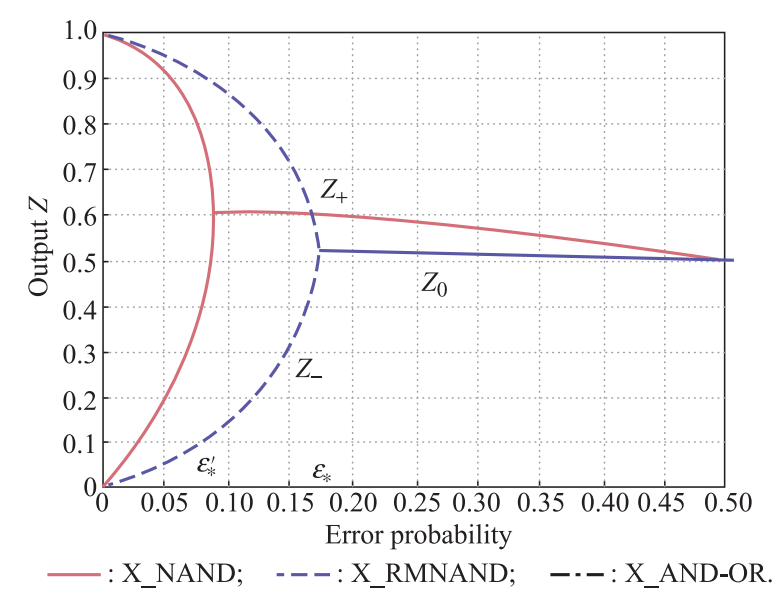

Fig. 6 Bifurcation diagram of XOR multiplexing system

As can be seen from Fig. 6, X_RMNAND has a larger error threshold than X_NAND and X_AND-OR. And X_AND-OR has exactly the same bifurcation diagram as the X_NAND. This means that although their components and iterative equations are different, they have the same gate error tolerant ability, and their abilities are much 
smaller than the ability of X_RMNAND. Obviously, compared to the NAND restoring organ and the AND-OR restoring organ, the redundancy-modified NAND restoring organ has a more superior fault tolerant performance. The analysis of the fault tolerant ability of X_RMNAND will be discussed in the next section.

\section{Fault tolerant ability analysis}

\subsection{Analysis of gate error threshold}

From Fig.6 we can see that the bifurcation points (error thresholds) divide the diagram into two regions, which are (i) $0<\varepsilon<\varepsilon_{*}\left(0<\varepsilon<\varepsilon_{*}^{\prime}\right)$ and (ii) $\varepsilon_{*}<\varepsilon<1 / 2$ $\left(\varepsilon_{*}^{\prime}<\varepsilon<1 / 2\right)$. By solving the equation

$$
Z_{0}=(1-\varepsilon)\left(1-Z_{0}^{2}\right)^{2}+\varepsilon\left[1-\left(1-Z_{0}^{2}\right)^{2}\right]
$$

one obtains $Z_{0}$, where $Z_{0}$ is the fixed point solution for interval $0<\varepsilon<1 / 2$.

In region (ii), $Z_{0}$ is a stable fixed point. By stable, it means that for any arbitrary initial inputs condition $X$ and $Y$, the output $Z_{n}$ will converge to $Z_{0}$ when $n$ is large, which means in this region, the system no longer functions. Expanding (12), we get a univariate quartic equation

$$
(1-2 \varepsilon) Z_{0}^{4}+(4 \varepsilon-2) Z_{0}^{2}-Z_{0}+1-\varepsilon=0
$$

which may be written as

$$
Z_{0}^{4}+A_{2} Z_{0}^{2}+A_{1} Z_{0}+A_{0}=0
$$

where $A_{2}=-2, A_{1}=\frac{-1}{1-2 \varepsilon}, A_{0}=\frac{1-\varepsilon}{1-2 \varepsilon}$. By solving (14), we obtain four roots, but two of them are imaginary roots, and the third root lies outside the range of [ [ 1 1], only the fourth root is the desired one. And the desired root of (14) is

$$
Z_{0}=\frac{\alpha-\sqrt{\alpha^{2}-4 t}}{2}
$$

where

$$
\begin{gathered}
t=\frac{A_{2}+\alpha^{2}+\frac{A_{1}}{\alpha}}{2} \\
\alpha=\sqrt{c_{0}-\frac{B_{1}}{3 c_{0}}-\frac{2 A_{2}}{3}} \\
c_{0}=\left[\left(-\frac{B_{0}}{2}+\frac{1}{2} \sqrt{B_{0}^{2}+\frac{4 B_{1}^{3}}{27}}\right)\right]^{\frac{1}{3}} \\
B_{0}=\frac{72 A_{0} A_{2}-2 A_{2}^{3}-27 A_{1}^{2}}{27} \\
B_{1}=\frac{-A_{2}^{2}-12 A_{0}}{3} .
\end{gathered}
$$

For further details, please refer to [30]. For X_NAND, when $\varepsilon_{*}^{\prime}<\varepsilon<1 / 2$, the system also has a stable fixed point solution, by solving the equation $z_{0}=1-\varepsilon+(2 \varepsilon-$ 1) $z_{0}^{2}$, then we get

$$
z_{0}=\frac{1-\sqrt{1-4(2 \varepsilon-1)(1-\varepsilon)}}{2(2 \varepsilon-1)} .
$$

Since X_AND-OR has exactly the same bifurcation diagram as X_NAND, the fixed point solution of X_AND-OR is (21) too.

When $0 \leqslant \varepsilon<\varepsilon_{*}, Z_{0}$ loses stability, and the motion is periodic with a period of 2 . We denote those two points by $Z_{+}$and $Z_{-}$. In this region, at the $n$th stage, when $Z_{+}$ is the input, then $Z_{-}$is the output and vice versa $[15,18]$. That means

$$
\begin{aligned}
& Z_{+}=(1-2 \varepsilon) Z_{-}^{4}+(4 \varepsilon-2) Z_{-}^{2}+1-\varepsilon \\
& Z_{-}=(1-2 \varepsilon) Z_{+}^{4}+(4 \varepsilon-2) Z_{+}^{2}+1-\varepsilon .
\end{aligned}
$$

Clearly, when $Z_{+}=Z_{-}$, we have $\varepsilon=\varepsilon_{*}$. By solving (22) and (23), it can be derived that $\varepsilon_{*}=0.17063$. Now it is easy to see that $0 \leqslant \varepsilon<\varepsilon_{*}$ is the parameter interval where the system functions and $\varepsilon_{*}$ is the gate error threshold. When $\varepsilon>\varepsilon_{*}$, the outputs converge to stable fixed point $Z_{0}$ regardless of what the initial inputs are. The gate error thresholds of X_NAND and X_AND-OR can be derived in the same way. They both are 0.08856 . Obviously, multiplexing architectures based on redundancy-modified NAND restoring organs can tolerate a device error rate of up to $10^{-1}$, while multiplexing architectures based on NAND restoring organs and AND-OR restoring organs can only tolerate a device error rate of up to $10^{-2}$. Properly speaking, the gate error tolerant ability of X_RMNAND is almost twice as much as that of X_NAND and X_AND$\mathrm{OR}$. There is no doubt that through the use of redundancymodified NAND restoring organs, the gate error tolerant ability of multiplexing has been greatly improved.

\subsection{Analysis of input signal error threshold}

In the last subsection, we analyze one aspect of fault tolerant ability of the system, namely, the tolerant ability for gate error. Now let us analyze the tolerant ability for input signal error. In order to map each output probability to a logic state, we need a threshold $z_{*}$. According to Fig. 6, a simple scheme is to set threshold at $z_{*}=Z_{0}\left(\varepsilon_{*}\right) . Z_{0}\left(\varepsilon_{*}\right)$ is a good approximation of $Z_{0}\left(\left|Z_{0}(\varepsilon)-Z_{0}\left(\varepsilon_{*}\right)\right| \leqslant 0.0043\right)$. However, in order to obtain more precise analysis, it is better to set $z_{*}=Z_{0}$ (and set $z_{*}^{\prime}=z_{0}$ for X_NAND and X_AND-OR).

Below, we shall interpret $\left[0, z_{*}\right)$ as non-stimulated state and $\left(z_{*}, 1\right]$ as stimulated state or $\left[0, z_{*}^{\prime}\right)$ as non-stimulated 
state and $\left(z_{*}^{\prime}, 1\right]$ as stimulated state for X_NAND and X_AND-OR

For X_RMNAND, fix the inputs $Y=1$ and $Y=0$, then we can get 3-D diagrams as showed in Fig. 7.

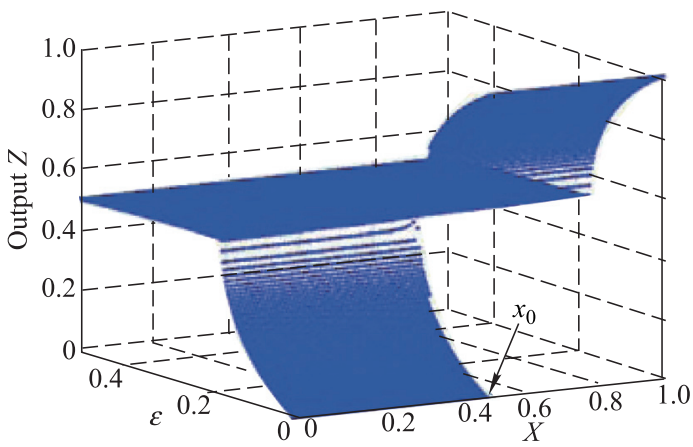

(a) Fixed $Y=0$

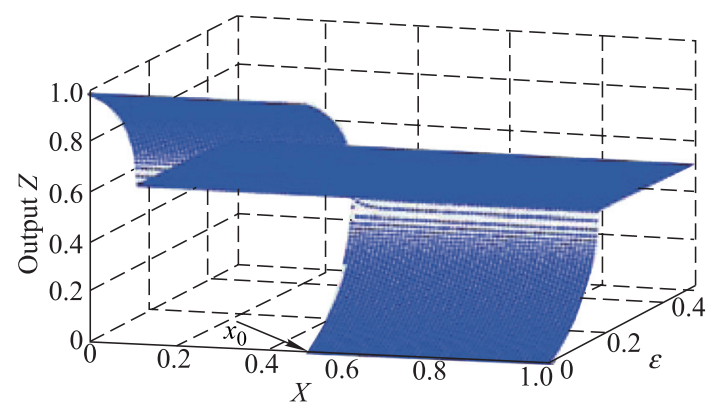

(b) Fixed $Y=1$

Fig.7 3-D diagrams of XOR multiplexing

A fact has been noticed that for each different fixed $Y$, when $0 \leqslant \varepsilon<\varepsilon_{*}$, there are different values of $X$ (here we call it as critical point and denoted by $x_{0}$ ) dividing the output into two states. Take $Y=1$ as an example, for $0 \leqslant \varepsilon<\varepsilon_{*}$, when $X<x_{0}$, the output would be stimulated and when $X>x_{0}$, the output would be non-stimulated. The calculation of the critical point can help us more intuitively understand the fault tolerant ability of the system. Now let us discuss the mathematic relation between them. When $n$ is large enough and $0 \leqslant \varepsilon<\varepsilon_{*}$, the output is only dependent on the input condition: input $X$ and input $Y$ have the same logic state (both stimulated or both nonstimulated) or have different logic states (one of the inputs is stimulated and the other one is non-stimulated). Let us denote the probability that $X$ and $Y$ have different logic states by $P_{1}$, and denote the probability that two inputs $X$ and $Y$ have the same logic state by $P_{2}$. Thus the ratios of $P_{1}$ and $P_{2}$ will be a key parameter to determine the final output $Z_{n}$ is larger than $z_{*}$ or not. $X$ and $Y$ are the probabilities of inputs being stimulated, then $1-X$ and $1-Y$ are the probabilities of inputs being non-stimulated. $P_{1}$ and $P_{2}$ are shown as follows:

$$
\begin{aligned}
& P_{1}=X(1-Y)+Y(1-X) \\
& P_{2}=X Y+(1-X)(1-Y) .
\end{aligned}
$$

If we need the output to be stimulated, then $P_{1} / P_{2}$ must be larger than a specific value which is greater than one. Since the output logic state is associated with the output threshold $z_{*}$, so the specific value would be a function of $z_{*}$ and the mathematic relation between them is shown as follows:

$$
\frac{P_{1}}{P_{2}}=\frac{X(1-Y)+Y(1-X)}{X Y+(1-X)(1-Y)}>\frac{z_{*}}{1-z_{*}} .
$$

Clearly, $P_{1}+P_{2}=1$, hence, (26) is equivalent to

$$
P_{1}=X(1-Y)+Y(1-X)>z_{*} .
$$

If $P_{1}>z_{*}$, the final output would be larger than the threshold (stimulated). When the inequality becomes

$$
P_{1}=X(1-Y)+Y(1-X)<z_{*}
$$

the final output would be smaller than the threshold (nonstimulated). Hence, it is easy to obtain the critical point $x_{0}$ by solving the equality

$$
x_{0}(1-Y)+Y\left(1-x_{0}\right)=z_{*} .
$$

X_NAND and X_AND-OR have the same property, and their critical point $x_{0}^{\prime}$ can be obtained by solving

$$
x_{0}^{\prime}(1-Y)+Y\left(1-x_{0}^{\prime}\right)=z_{*}^{\prime} .
$$

Clearly, the critical point is a function of $Y$ and $\varepsilon$. Since when $\varepsilon_{*}<\varepsilon<1 / 2$, the system no longer functions, we only consider the region of $0 \leqslant \varepsilon \leqslant \varepsilon_{*}$. Threshold $z_{*}\left(z_{*}^{\prime}\right)$ changes monotonically with each different gate error probability $\varepsilon$, here, we choose the minimum $\varepsilon=0$ and the maximum $\varepsilon=\varepsilon_{*}$ as representatives. Plot those corresponding critical points against each $Y(\Delta Y=0.01)$, this lead to Fig. 8.

Just like the bifurcation diagram, X_NAND and X_AND-OR have exactly the same critical points distribution. Fig. 8 shows that there is slight difference between critical points corresponding to $\varepsilon=0$ and those corresponding to $\varepsilon=\varepsilon_{*}$. And the difference of X_NAND and X_AND-OR is more obvious than the difference of X_RMNAND. This indicates that compared to X_NAND and X_AND-OR, the critical points of X_RMNAND are less affected by the gate error probability $\varepsilon$. As we can see, for each different $Y$, the critical point has different values and there is a parameter interval that makes the system no longer function even though the system is fault free $(\varepsilon=0)$. If the stimulated probability of one of the 
inputs is in this interval, then the outputs will always be non-stimulated even though the other input is $100 \%$ being stimulated or non-stimulated, namely, the output is stuck at 0 .

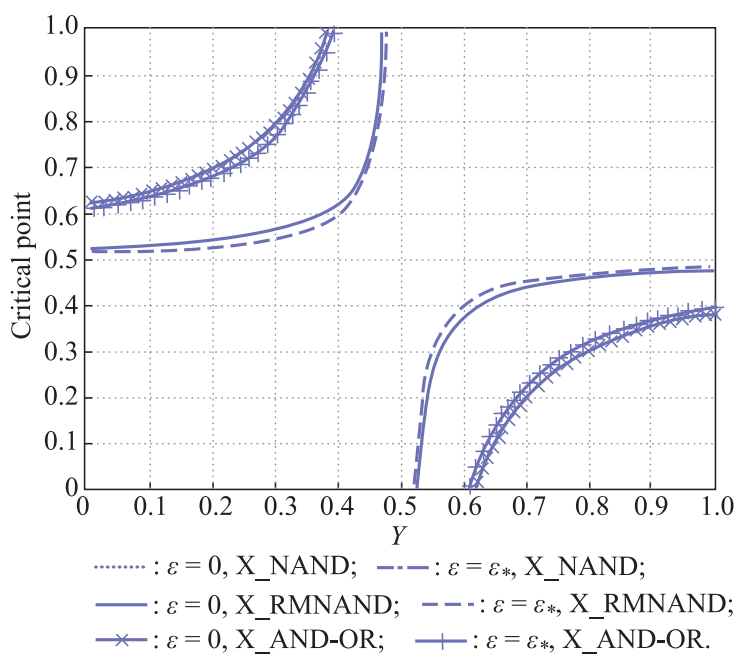

Fig. 8 Critical point against each $Y$
For more rigorous analysis of the results, we analyze the maximum interval that corresponds to $\varepsilon=0$. This parameter interval of X_RMNAND is about $0.4751<Y<$ 0.5249 . Thus, the invalid input signal stimulation probability interval occupies only $4.98 \%$. While the parameter interval of X_NAND and X_AND-OR is about $0.3820<$ $Y<0.6180$, and the invalid input signal stimulation probability interval occupies $23.6 \%$, which is much larger than $4.98 \%$. This means that the X_RMNAND has a much better input signal error tolerant ability than X_NAND and X_AND-OR. To be more specific, compared to X_NAND and X_AND-OR, the invalid input signal stimulation probability interval of X_RMNAND is reduced by $78.898 \%$. There is no doubt that this is a significant improvement in input signal error tolerant ability.

In order to more intuitively understand tolerant ability for input signal error of the system, several fixed $Y$ and the corresponding critical points are extracted from Fig. 8. These lead to Table 1, obviously, X_RMNAND can tolerate much more input signal error than X_NAND and X_ANDOR do.

Table 1 Critical points for several fixed $Y(\varepsilon=0)$

\begin{tabular}{ccccccccccc}
\hline$Y$ & 0 & 0.1 & 0.2 & 0.3 & 0.4 & 0.6 & 0.7 & 0.8 & 0.9 & 1.0 \\
\hline$x_{0}$ & 0.5249 & 0.5311 & 0.5415 & 0.5622 & 0.6244 & 0.3756 & 0.4378 & 0.4585 & 0.4689 & 0.4751 \\
$x_{0}^{\prime}$ & 0.6180 & 0.6475 & 0.6967 & 0.7951 & $/$ & $/$ & 0.2049 & 0.3033 & 0.3525 & 0.3820 \\
\hline
\end{tabular}

Assume that a stimulated output is expected, and then two inputs should have different logic states. Let us take $Y=0.7$ as an example, in X_RMNAND, it can be seen that when input $Y$ has a probability of $70 \%$ being stimulated, any stimulated probability smaller than $43.78 \%$ of the other input $X$ could be accepted, namely, any nonstimulated probability not larger than $56.22 \%$ of input $X$ will work for the system. This means that the system can tolerate input signal error probabilities of $30 \%$ and $43.78 \%$ for the inputs $Y$ and $X$. X_NAND and X_AND-OR can only tolerate error probabilities of $30 \%$ and $20.49 \%$ for the inputs $Y$ and $X$. This indicates that in the case of tolerating error probability of $30 \%$ for input $Y$, the tolerant ability of X_RMNAND for the other input signal $X$ is about 2.1366 times that of X_NAND and X_AND-OR.

The emerging nanometer-scale devices are harder to fabricate and more sensitive to external influences. This makes nano-meter devices more prone to transient faults than complementary metal oxide semiconductor (CMOS) devices. The same as NAND multiplexing, although the XOR multiplexing technique is efficient against the increasing transient faults, it is less efficient against manufacturing defects or permanent faults. And because of the high redundancy, it may be more suitable for high hardware cost applications rather than low hardware cost applications.

\section{Conclusions}

In order to make systems based on nanoelectronic devices reliable, the design of fault tolerant architectures will be necessary. This paper can be seen as a part of the endeavor devoted to this work. In this paper, we study multiplexing architectures based on redundancy-modified NAND restoring organs and compare it to two other multiplexing architectures based on traditional NAND restoring organs and two layers AND-OR restoring organs, respectively. Experimental results show that this new fault tolerant architecture has a much higher fault tolerant ability of gate error probability and input signal error probability. This architecture is potentially effective in protection against the increasing transient faults for systems based on unreliable nanometer-scale devices.

\section{References}

[1] ANJANKAR S C, KOLTE DR M T, PUND A, et al. FPGA based multiple fault tolerant and recoverable technique using triple modular redundancy (FRTMR). Procedia Computer Science, 2016, 79: $827-834$

[2] YAO R, CHEN Q, LI Z W, et al. Multi-objective evolutionary design of selective triple modular redundancy systems against SEUs. Chinese Journal of Aeronautics, 2015, 28(3): 804-813. 
[3] CHEN Y K, FENG Z G, ZHANG S, et al. Research on reconfigurable triple-module redundancy space on-board computer. Computer Measurement \& Control, 2017, 25(2): 201-203. (in Chinese)

[4] SIOZIOS K, SAVIDIS I, SOUDRIS D. A framework for exploring alternative fault-tolerant schemes targeting 3-D reconfigurable architectures. Proc. of the International Conference on Embedded Computer Systems: Architectures, Modeling and Simulation, 2016: 336-341.

[5] HE R X, CHEN N J. Fault tolerance of reconfigurable computing system. Journal of Mianyang Teachers'College, 2017, 36(5): 90 - 97. (in Chinese)

[6] BANERJEE S, RAO W. A local reconfiguration based scalable fault tolerant many-processor array. Proc. of the 22nd Asia and South Pacific Design Automation Conference, 2017: 432 437.

[7] HAN J, LEUNG E, LIU L B, et al. A fault-tolerant technique using quadded logic and quadded transistors. IEEE Trans. on Very Large Scale Integration Systems, 2015, 23(8): 1562 1566.

[8] ROHANIPOOR M R, GHAVAMI B, RAJI M. Design of fault tolerant digital integrated circuits based on quadded transistor logic. Proc. of the 8th International Conference on Information and Knowledge Technology, 2016: $188-192$.

[9] ZHANG J B, CAI J Y, MENG Y F. A design technology of fault tolerance circuit system facing complex electromagnetic environments. Journal of Xi'an Jiaotong University, 2017, 51(2): 53-59. (in Chinese)

[10] SOLOVIEV A, STEMPKOVSKY A, KALEEV D, et al. Methods of increasing the fault-tolerance of control unit by introducing hardware redundancy. Proc. of the IEEE Internet Technologies and Applications, 2015: 37-40.

[11] SHOKER A. Exploiting universal redundancy. Proc. of the 15th IEEE International Symposium on Network Computing and Applications, 2016: 199-203.

[12] KIM J, LEE W, CHO K, et al. Hardware-efficient built-in redundancy analysis for memory with various spares. IEEE Trans. on Very Large Scale Integration Systems, 2017, 25(3): $844-856$.

[13] SHEIKH A T, EL-MALEH A H, ELRABAA M E S, et al. A fault tolerance technique for combinational circuits based on selective-transistor redundancy. IEEE Trans. on Very Large Scale Integration Systems, 2017, 25(1): 224-237.

[14] SUN Q, NIE L, SUN L, et al. Hybrid redundancy fault tolerant codec in distributed storage system. Proc. of the International Conference on Industrial Informatics, 2015: 22-25.

[15] QI Y, GAO J B, FORTES A B. Markov chains and probabilistic computation-a general framework for multiplexed nanoelectronic systems. IEEE Trans. on Nanotechnology, 2005, 4(2): $194-205$.

[16] VON NEUMANN J. Probabilistic logics and the synthesis of reliable organisms from unreliable components. Princeton: Princeton University Press, 1956.

[17] PIPPENGER N. Invariance of complexity measures for networks with unreliable gates. Journal of the ACM, 1989, 36(3): $531-539$.

[18] QI Y, GAO J B. Bifurcations and fundamental error bounds for fault tolerant computations. IEEE Trans. on Nanotechnology, 2005, 4(4): $395-402$.

[19] HAN J, JONKER P. A system architecture solution for unreliable nanoelectronic devices. IEEE Trans. on Nanotechnology, 2002, 1(4): $201-208$.

[20] VOICU G R, COTOFANA S D. Towards heterogenous 3Dstacked reliable computing with von Neumann multiplexing. Proc. of the IEEE/ACM International Symposium on
Nanoscale Architectures, 2013: 122-127.

[21] GUCWA K. On simulation of multiplexed architecture for fault-tolerant nanoelectronic systems. Proc. of the 12th IEEE Conference on Nanotechnology, 2012: 1-4.

[22] BEIU V, IBRAHIM W. Devices and input vectors are shaping von Neumann multiplexing. IEEE Trans. on Nanotechnology, 2011, 10(3): 606-616.

[23] LEE J S, JEON J C. Design of low hardware complexity multiplexer using NAND gates on quantum-dot cellular automata. International Journal of Multimedia and Ubiquitous Engineering, 2016, 11(12): $307-318$.

[24] ROY S, BEIU V. Majority multiplexing-economical redundant fault-tolerant designs for nanoarchitectures. IEEE Trans. on Nanotechnology, 2005, 4(4): $441-451$.

[25] BHADURI D, SHUKLA S K. Reliability evaluation of von Neumann multiplexing based defect-tolerant majority circuits. Proc. of the 4th IEEE Conference on Nanotechnology, 2004: $599-601$.

[26] BHADURI D, SHUKLA S K, GRAHAM P, et al. Comparing reliability-redundancy tradeoffs for two von Neumann multiplexing architectures. IEEE Trans. on Nanotechnology, 2007, 6(3): $265-279$.

[27] IBRAHIM W, BEIU V, BEG A. On NOR-2 von Neumann multiplexing. Proc. of the 5th International Design and Test Workshop, 2010: 67-72.

[28] PIERCE W H. Interwoven redundant logic. Journal of the Franklin Institute, 1964, 277(1): 55-85.

[29] DIAO M, YU L H, CHEN X B. A fault tolerant technique for naocomputers: XOR multiplexing. ICIC Express Letters, 2017, 11(7): $1167-1174$.

[30] MAHMOOD M, HAMMAD S, MAHMOOD I. An efficient algorithm for computing the roots of general quadratic, cubic and quartic equations. International Journal of Mathematical Education in Science and Technology, 2014, 45(7): $1612-$ 1620.

\section{Biographies}

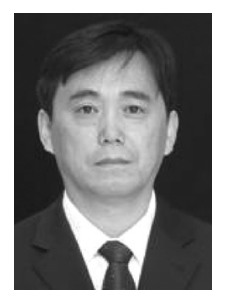

DIAO Ming was born in 1960. He received his B.S. degree in communication and information system from Harbin Engineering University, Harbin, China, in 1987. He is currently a senior member of China Communications Institute, a member of China Society of Image and Graphics, a member of explorations professional committee of China Aerospace Society and a professor and doctoral supervisor with the Harbin Engineering University, China. He is also an editorial board member of Journal of Harbin Engineering University. His current research interests include the detection, processing and recognition of broadband signal and communication signal processing. He has been involved in more than 10 provincial scientific research projects and published over a hundred academic papers.

E-mail: diaoming@hrbeu.edu.cn

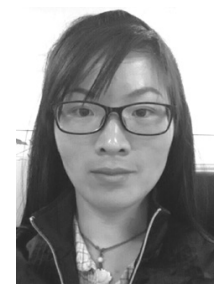

YU Lianhua was born in 1989. She received her B.E. degree in electronic information engineering from Harbin Engineering University, Harbin, China, in 2012. She is currently working toward her Ph.D. degree in information and communication engineering at Harbin Engineering University. Her major research interests include fault-tolerant computation, reliable computing with unreliable components, and nanoelectronic system architectures.

E-mail: ruying0714@hotmail.com 


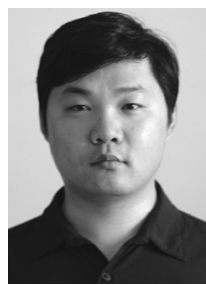

CHEN Xiaobo was born in 1989. He received his B.E. degree in communication engineering from Harbin Engineering University, Harbin, China, in 2011. He is currently working toward his Ph.D. degree in navigation, guidance and control at Harbin Engineering University. His research interests include navigation systems, nonlinear control systems and applications, formation control, and signal processing.

E-mail: cxiaobo@hotmail.com 